\title{
Elliptic Means and Their Generalizations
}

\author{
By \\ Peter Kahlig and Janusz Matkowski \\ (Vorgelegt in der Sitzung der math.-nat. Klasse am 9. Oktober 2003 \\ durch das w. M. Ludwig Reich)
}

\begin{abstract}
In this note we introduce a one-parameter family of homogeneous means strictly related to ellipses. Each member of the family is a weighted power mean, and only one of them is both symmetric and quasi-arithmetic. Geometric interpretations are given, and higher-dimensional counterparts of these means are defined. Iterations of some mean-type mappings and some functional equations are considered.
\end{abstract}

1991 Mathematics Subject Classification: Primary 26E60, Secondary 26A18.

Key words and phrases: Elliptic mean, ellipsoidal mean, iteration of mean-type mapping, invariant mean, functional equation.

\section{Introduction}

Let $I \subset \mathbb{R}$ be an interval and $k \in \mathbb{N}, k \geq 2$, fixed. A function $M: I^{k} \rightarrow \mathbb{R}$ is said to be a mean if

$$
\min \left(x_{1}, \ldots, x_{k}\right) \leq M\left(x_{1}, \ldots, x_{k}\right) \leq \max \left(x_{1}, \ldots, x_{k}\right), \quad x_{1}, \ldots, x_{k} \in I .
$$

A mean $M$ is called strict if $\min \left(x_{1}, \ldots, x_{k}\right)<\max \left(x_{1}, \ldots, x_{k}\right)$ implies that the above inequalities are strict, and $M$ is called symmetric if, for every permutation $\sigma:\{1, \ldots, k\} \rightarrow\{1, \ldots, k\}$,

$$
M\left(x_{1}, \ldots, x_{k}\right)=M\left(x_{\sigma(1)}, \ldots, x_{\sigma(k)}\right), \quad x_{1}, \ldots, x_{k} \in I .
$$

For any continuous and strictly monotonic function $\varphi: I \rightarrow \mathbb{R}$ and a sequence $w=\left(w_{1}, \ldots, w_{k}\right), \quad w_{1}>0, \ldots, w_{k}>0, \quad w_{1}+\cdots$ 
$+w_{k}=1$, the function $M_{k, w}^{\varphi}: I^{k} \rightarrow \mathbb{R}$,

$$
M_{k, w}^{\varphi}\left(x_{1}, \ldots, x_{k}\right):=\varphi^{-1}\left(w_{1} \varphi\left(x_{1}\right)+\cdots+w_{k} \varphi\left(x_{k}\right)\right), \quad x_{1}, \ldots, x_{k} \in I,
$$

is a strict mean, and it is called a weighted quasi-arithmetic mean. The function $\varphi$ is referred to as a generator of the mean $M_{k, w}^{\varphi}$ and the numbers $w_{1}, \ldots, w_{k}$ as its weights. $\boldsymbol{M}_{k, w}^{\varphi}$ is symmetric iff $\boldsymbol{M}_{k, w}^{\varphi}=M_{k}^{\varphi}$ where

$$
M_{k}^{\varphi}\left(x_{1}, \ldots, x_{k}\right):=\varphi^{-1}\left(\frac{\varphi\left(x_{1}\right)+\cdots+\varphi\left(x_{k}\right)}{k}\right), \quad x_{1}, \ldots, x_{k} \in I,
$$

and $M_{k}^{\varphi}$ is called a quasi-arithmetic mean

A mean $M:(0, \infty)^{k} \rightarrow(0, \infty)$ is called homogeneous if

$$
M\left(t x_{1}, \ldots, t x_{k}\right)=t M\left(x_{1}, \ldots, x_{k}\right), t, x_{1}, \ldots, x_{k}>0 .
$$

It is well known (cf. B. JESSEN [4], also G. H. HARDY, J. E. LITTLEWOOD and G. PÓLYA [2], p. 68) that a weighted quasi-arithmetic mean is homogeneous iff it is a weighted power mean, that is, there is an $r \in \mathbb{R}$ such that $M_{k, w}^{[\varphi]}=M_{k, w}^{[r]}$ where

$$
M_{k, w}^{[r]}\left(x_{1}, \ldots, x_{k}\right):= \begin{cases}\left(w_{1} x_{1}^{r}+\cdots+w_{k} x_{k}^{r}\right)^{1 / r} & \text { for } r \neq 0, \\ x_{1}^{w_{1}} \cdots x_{k}^{w_{k}} & \text { for } r=0 .\end{cases}
$$

Note that $M_{k}^{[0]}=G_{k}$ where $G_{k}$ denotes the geometric mean.

In this note we show that the means $M_{2, w}^{[-2]}$ are strictly related to an ellipse, $M_{3, w}^{[-2]}$ to an ellipsoid, and $M_{k, w}^{[-2]}$ to a $k$-dimensional ellipsoid. In the first four sections we distinguish them by suitable symbols and formulate some of their properties as Propositions. In Section 5 we apply these Propositions to find all continuous solutions of a functional equation involving these means, closely related to the iteration of mean-type mappings.

\section{Elliptic Means}

We begin this section with the following quickly verifiable

Remark 1. Let $p>0$ be fixed. Then the function $E_{p}:(0, \infty)^{2} \rightarrow$ $(0, \infty)$,

$$
E_{p}(a, b):=a b \sqrt{\frac{p^{2}+1}{p^{2} a^{2}+b^{2}}}
$$

is a mean. 
We call the means $E_{p}$ elliptic which is justified by the following

Geometric Interpretation. Consider an ellipse given by the equation

$$
\frac{x^{2}}{a^{2}}+\frac{y^{2}}{b^{2}}=1
$$

where $a, b>0$, and take an arbitrary $p>0$. It may be shown that the length $|O P|$, where $O$ is the center of the ellipse and $P$ is the intersection point of the ellipse and the half-line

$$
y=p x, \quad x \geq 0,
$$

is given by $|O P|=E_{p}(a, b)$.

\section{Proposition 1.}

1. For every $p>0, E_{p}$ is a weighted power mean $M_{2, w}^{[-2]}$ with weights

$$
w=\left(\frac{1}{p^{2}+1}, \frac{p^{2}}{p^{2}+1}\right)
$$

in particular, it is homogeneous, and the function $\varphi(t)=t^{-2}(t>0)$ is a generator of this mean.

2. $E_{p}$ is symmetric iff $p=1$;

3. $E_{p}$ is quasi-arithmetic iff $p=1$; moreover,

$$
E_{1}=M_{2}^{[-2]} \text {. }
$$

4. For every $p>0, E_{p}$ is $G_{2}$-conjugate to the weighted square-root mean $M_{2, w}^{[2]} *$ with weights

$$
w^{*}=\left(\frac{p^{2}}{p^{2}+1}, \frac{1}{p^{2}+1}\right),
$$

that is

$$
G_{2} \circ\left(E_{p}, M_{2, w^{*}}^{[2]}\right)=G_{2},
$$

where $G_{2}$ denotes the geometric mean. $G_{2}$ is the unique continuous mean which is invariant with respect to the mean-type mapping $\left(E_{p}, M_{2, w^{*}}^{[2]}\right):(0, \infty)^{2} \rightarrow(0, \infty)^{2} ;$ moreover, the sequence of iterates of the mean-type mapping $\left(E_{p}, M_{2, w^{*}}^{[2]}\right)$ converges to the meantype mapping $\left(G_{2}, G_{2}\right)$ in $(0, \infty)^{2}$.

5. $\lim _{p \rightarrow 0} E_{p}(x, y)=x$ and $\lim _{p \rightarrow \infty} E_{p}(x, y)=y$ for all $x, y>0$. 
Proof. Parts 1-3 and 5 are not hard to verify. Part 4 is a consequence of some more general facts (the conjugate and invariant means were considered in [5] and [6], cf. also [1]).

Remark 2. It can be readily shown that the following commutation relation (involving a parameter transformation) holds:

$$
E_{p}(a, b)=E_{1 / p}(b, a), \quad p, a, b>0 .
$$

\section{Ellipsoidal Means}

Let $p, q>0$ be fixed. Then the function $E_{p, q}:(0, \infty)^{3} \rightarrow(0, \infty)$,

$$
E_{p, q}(a, b, c):=a b c \sqrt{\frac{p^{2}+q^{2}+1}{b^{2} c^{2}+p^{2} c^{2} a^{2}+q^{2} a^{2} b^{2}}}
$$

is a mean. $E_{p, q}$ can be called an ellipsoidal mean because of the following

Geometric Interpretation. Consider an ellipsoid given by the equation

$$
\frac{x^{2}}{a^{2}}+\frac{y^{2}}{b^{2}}+\frac{z^{2}}{c^{2}}=1
$$

where $a, b, c>0$, and take the half-line determined by the equations

$$
y=p x, \quad z=q x, \quad x \geq 0,
$$

for some arbitrary $p, q>0$. Calculations show that $E_{p, q}(a, b, c)$ is the length $|O P|$ where $O$ is the center of the ellipsoid and $P$ is the point of intersection of the ellipsoid and the half-line.

\section{Proposition 2.}

1. For all $p, q>0, E_{p, q}$ is a weighted power mean $M_{3, w}^{[-2]}$ with weights

$$
w=\left(\frac{1}{p^{2}+q^{2}+1}, \frac{p^{2}}{p^{2}+q^{2}+1}, \frac{q^{2}}{p^{2}+q^{2}+1}\right) ;
$$

in particular, it is homogeneous, and the function $\varphi(t)=t^{-2}$ $(t>0)$ is a generator of this mean.

2. $E_{p, q}$ is symmetric iff $p=q=1$;

3. $E_{p, q}$ is quasi-arithmetic iff $p=q=1$; moreover,

$$
E_{1,1}=M_{3}^{[-2]} \text {. }
$$


4. $G_{3}$ is the unique continuous mean which is invariant with respect to the mean-type mapping $\left(E_{p, q}, K_{p, q}, M_{3, w^{*}}\right):(0, \infty)^{3} \rightarrow(0, \infty)^{3}$ where the mean $K_{p, q}:(0, \infty)^{3} \rightarrow(0, \infty)$ is defined by

$$
\begin{gathered}
K_{p, q}(a, b, c):=\sqrt{\frac{p^{2} b^{2} c^{2}+q^{2} a^{2} b^{2}+c^{2} a^{2}}{p^{2} b^{2}+q^{2} a^{2}+c^{2}}}, \\
w^{*}=\left(\frac{q^{2}}{p^{2}+q^{2}+1}, \frac{p^{2}}{p^{2}+q^{2}+1}, \frac{1}{p^{2}+q^{2}+1}\right)
\end{gathered}
$$

that is

$$
G_{3} \circ\left(E_{p, q}, K_{p, q}, M_{3, w^{*}}^{[2]}\right)=G_{3} ;
$$

the sequence of iterates of the mean-type mapping $\left(E_{p, q}, K_{p, q}, M_{3, w^{*}}^{[2]}\right)$ converges to the mean-type mapping $\left(G_{3}, G_{3}, G_{3}\right)$ in $(0, \infty)^{3}$.

Moreover, $K_{p, q}$ is symmetric iff $p=q=1$.

5. $\lim _{q \rightarrow 0} E_{p, q}(x, y, z)=E_{p}(x, y)$ and $\lim _{q \rightarrow \infty} E_{p, q}(x, y, z)=z$ for all $x, y, z>0$.

Remark 3. The following commutation relations (involving some parameter transformations) can be readily verified:

$$
\begin{aligned}
E_{p, q}(a, b, c) & =E_{q, p}(a, c, b)=E_{1 / p, q / p}(b, a, c)=E_{q / p, 1 / p}(b, c, a) \\
& =E_{p / q, 1 / q}(c, b, a)=E_{1 / q, p / q}(c, a, b)
\end{aligned}
$$

for all $a, b, c, p, q>0$.

\section{The General $k$-Dimensional Case}

Let $k \in \mathbb{N}, k \geq 2$, and $p_{1}, \ldots, p_{k-1}>0$ be fixed. Then the function $E_{p_{1}, \ldots, p_{k-1}}:(0, \infty)^{k} \rightarrow(0, \infty)$ defined by

$$
\begin{aligned}
& E_{p_{1}, \ldots, p_{k-1}}\left(a_{1}, \ldots, a_{k}\right) \\
& :=a_{1} \cdots a_{k} \sqrt{\frac{p_{1}^{2}+\cdots+p_{k-1}^{2}+1}{p_{1}^{2} a_{2}^{2} \cdots a_{k}^{2}+\cdots+p_{k-1}^{2} a_{1}^{2} \cdots a_{k-2}^{2} a_{k}^{2}+a_{1}^{2} \cdots a_{k-1}^{2}}} \\
& \times\left(E_{p_{1}, \ldots, p_{k-1}}\left(a_{1}, \ldots, a_{k}\right):=\left(\prod_{i=1}^{k} a_{i}\right) \sqrt{\frac{\sum_{i=1}^{k-1} p_{i}^{2}+1}{\sum_{j=1}^{k-1} p_{j}^{2} \prod_{i \neq j}^{k} a_{i}^{2}+\prod_{i=1}^{k-1} a_{i}^{2}}}\right)
\end{aligned}
$$

is a mean. This mean may be referred to as $k$-dimensional ellipsoidal mean by an analogous geometric interpretation as in the previous cases. 


\section{Proposition 3.}

1. For all $p_{1}, \ldots, p_{k-1}>0, E_{p_{1}, \ldots, p_{k-1}}$ is a weighted power mean $M_{k, w}^{[-2]}$ with weights

$$
w=\left(\frac{1}{\sum_{i=1}^{k-1} p_{i}^{2}+1}, \frac{p_{1}^{2}}{\sum_{i=1}^{k-1} p_{i}^{2}+1}, \ldots, \frac{p_{k-1}^{2}}{\sum_{i=1}^{k-1} p_{i}^{2}+1}\right) ;
$$

in particular, it is homogeneous, and the function $\varphi(t)=t^{-2}$ $(t>0)$ is a generator of this mean.

2. $E_{p_{1}, \ldots, p_{k-1}}$ is symmetric iff $p_{1}=\cdots=p_{k-1}=1$;

3. $E_{p_{1}, \ldots, p_{k-1}}$ is quasi-arithmetic iff $p_{1}=\cdots=p_{k-1}=1$; moreover,

$$
E_{1, \ldots, 1}=M_{k}^{[-2]} .
$$

4. $G_{k}$ is the unique continuous mean which is invariant with respect to the mean-type mapping $\left(E_{1, \ldots, 1}, K_{1}, \ldots, K_{k-1}\right):(0, \infty)^{k} \rightarrow(0, \infty)^{k}$ where the means $K_{l}:(0, \infty)^{k} \rightarrow(0, \infty), \quad l=1, \ldots, k-1$, are defined by

$$
K_{l}\left(a_{1}, \ldots, a_{k}\right):=\sqrt{\frac{(k-l) \sum_{j_{1}<\cdots<j_{l}} \prod_{i \notin\left\{j_{1}, \ldots, j_{l}\right\}} a_{i}^{2}}{(l+1) \sum_{j_{1}<\cdots<j_{l+1}} \prod_{i \notin\left\{j_{1}, \ldots, j_{l+1}\right\}} a_{i}^{2}}},
$$

that is

$$
G_{k} \circ\left(E_{1, \ldots, 1}, K_{1}, \ldots, K_{k-1}\right)=G_{k} ;
$$

moreover, $K_{k-1}=M_{k}^{[2]}$, and the sequence of iterates of the meantype mapping $\left(E_{1, \ldots, 1}, K_{1}, \ldots, K_{k-2}, M_{k}^{[2]}\right)$ converges to the meantype mapping $\left(G_{k}, \ldots, G_{k}\right)$ in $(0, \infty)^{k}$.

\section{An Application to a Functional Equation}

HARUKI and RASSIAS [3] (cf. also [8]) posed the following

Problem 1. Is it true that every continuous function $f:(0, \infty) \times$ $(0, \infty) \rightarrow \mathbb{R}$ satisfying the functional equation

$$
f\left(\frac{2 x y}{x+y}, \frac{x+y}{2}\right)=f(x, y), \quad x, y>0,
$$

is of the form

$$
f(x, y)=F(x y), \quad x, y>0,
$$

where $F:(0, \infty) \rightarrow \mathbb{R}$ is a continuous function of a single variable? 
The affirmative answer has been given by the second author (cf. [7]). Functional equations of the form

$$
f(M(x, y), N(x, y))=f(x, y), \quad x, y \in I,
$$

where $M, N: I^{2} \rightarrow I$ are means in an interval $I$, play an essential role in some problems connected with iterations of means. For

$$
M(x, y):=\frac{x+y}{2} \text { and } N(x, y):=\sqrt{x y},
$$

this equation appears in connection with the $A G M$ iteration of Gauss and elliptic integrals (cf. for instance [1]).

Applying our Proposition 1 we can prove the following

Theorem 1. Let $p>0$ be arbitrarily fixed. Suppose that $f:(0, \infty)^{2} \rightarrow \mathbb{R}$ is continuous on the diagonal $\Delta:=\{(x, x): x>0\}$. Then $f$ satisfies the functional equation

$$
f\left(x y \sqrt{\frac{p^{2}+1}{p^{2} x^{2}+y^{2}}}, \sqrt{\frac{p^{2} x^{2}+y^{2}}{p^{2}+1}}\right)=f(x, y), \quad x, y>0,
$$

if, and only if,

$$
f(x, y)=F(x y), \quad x, y>0,
$$

where $F:(0, \infty) \rightarrow \mathbb{R}$ is a continuous function of a single variable.

Proof. Suppose that $f:(0, \infty) \times(0, \infty) \rightarrow \mathbb{R}$ is continuous and satisfies equation (1). Since

$$
E_{p}(x, y)=x y \sqrt{\frac{p^{2}+1}{p^{2} x^{2}+y^{2}}}, \quad M_{2, w^{*}}^{[2]}(x, y)=x y \sqrt{\frac{p^{2} x^{2}+y^{2}}{p^{2}+1}}, \quad x, y>0,
$$

we can write equation (1) in the form

$$
f \circ\left[\left(E_{p}, M_{2, w^{*}}^{[2]}\right)\right]=f .
$$

Hence, by induction, we get

$$
f \circ\left[\left(E_{p}, M_{2, w^{*}}^{[2]}\right)^{n}\right]=f, \quad n \in \mathbb{N},
$$

where $\left(E_{p}, M_{2, w^{*}}^{[2]}\right)^{n}$ denotes the $n$-th iteration of the mean-type mapping $\left(E_{p}, M_{2, w^{*}}^{[2]}\right)$. By Proposition 1 , letting here $n \rightarrow \infty$ and making use of the continuity of $f$ on $\Delta$, we obtain

$$
f \circ\left[\left(G_{2}, G_{2}\right)\right]=f,
$$


that is

$$
f(x, y)=f[(\sqrt{x y}, \sqrt{x y})], \quad x, y>0 .
$$

Setting

$$
F(u):=f(\sqrt{u}, \sqrt{u}), \quad u>0,
$$

we have

$$
f(x, y)=F(x y), \quad x, y>0 .
$$

Since the converse implication requires only simple calculations, the proof is complete.

Similarly, applying Proposition 2, we can prove

Theorem 2. Let $p, q>0$ be fixed. Suppose that $f:(0, \infty)^{3} \rightarrow \mathbb{R}$ is continuous on the diagonal $\Delta:=\{(x, x, x): x>0\}$. Then $f$ satisfies the functional equation

$$
f \circ\left(E_{p, q}(x, y, z), K_{p, q}(x, y, z), M_{3, w^{*}}^{[2]}(x, y, z)\right)=f(x, y, z), \quad x, y, z>0,
$$

if, and only if,

$$
f(x, y, z)=F(x y z), \quad x, y, z>0,
$$

where $F:(0, \infty) \rightarrow \mathbb{R}$ is a continuous function of a single variable.

Remark 4. A k-dimensional counterpart of the above results is also true. Its version for the symmetric means results from Proposition 3.

\section{Acknowledgement}

The authors appreciate critical comments of Professor Ludwig Reich.

\section{References}

[1] Borwein, J. M., Borwein, P. B. (1987) Pi and the AGM: A Study in Analytic Number Theory and Computational Complexity. Wiley, New York

[2] Hardy, G. H., Littlewood, J. E., Pólya, G. (1934) Inequalities. Cambridge University Press, Cambridge

[3] Haruki, H., Rassias, TH. M. (1995) A new analogue of Gauss' functional equation. Internat. J. Math. Sci. 18: 749-756

[4] JESSEN, B. (1931) Bemaerkninger om konvekse Funktioner og Uligheder imellem Middelvaerdier I, II. Mat. Tidsskr. B: 17-28, 84-95

[5] MatkowsKi, J. (1999) Invariant and complementary means. Aequationes Math. 57: $87-107$ 
[6] MatKowsKI, J. (1999) Iterations of mean-type mappings and invariant means. Ann. Math. Siles. 13: 211-226

[7] Matkowski, J. (2001) A solution of a problem of H. Haruki and Th. M. Rassias. Ann. Math. Siles. 15: 94

[8] SAHOO, P., RIEDEL, T. (1998) Mean Value Theorems and Functional Equations. World Scientific Publishing Co., Singapore

Authors' addresses: Peter Kahlig, c/o Institute of Meteorology and Geophysics, University of Vienna, UZA2, 1090 Vienna, Austria, E-Mail: Peter.Kahlig@univie. ac.at; Janusz Matkowski, Institute of Mathematics, University of Zielona Góra, Podgórna 50, 65246 Zielona Góra, Poland, E-Mail: J.Matkowski@im.uz.zgora.pl 